\title{
Glyceria spicata subsp. onubensis (Gramineae), nuevo taxon para la flora ibérica
}

\author{
Josefa López ${ }^{1} \&$ Juan Antonio Devesa² \\ ${ }^{1}$ Área de Botánica, Facultad de Ciencias, Universidad de Extremadura, Avenida de Elvas, s.n., 06006 Badajoz, España. \\ ${ }^{2}$ Departamento de Botánica, Ecología y Fisiología Vegetal, Facultad de Ciencias, Universidad de Córdoba, Campus de \\ Rabanales, Edificio José Celestino Mutis, Ctra. de Madrid km. 396 A, 14014 Córdoba, España.
}

\author{
Correspondencia \\ J. López \\ e-mail: josefalopez@unex.es \\ Recibido: 30 septiembre 2019 \\ Aceptado: 7 octubre 2019 \\ Publicado on-line: 10 octubre 2019 \\ Editado por: B. Cabezudo
}

Glyceria spicata subsp. onubensis (Gramineae), new taxon for the iberian flora

Palabras claves: Glyceria, Gramineae, Península Ibérica, Taxonomía.

Key words: Glyceria, Gramineae, Iberian Peninsula, Taxonomy.
El género Glyceria $\mathrm{R}$. Br. comprende entre 48 (Kellogg, 2015) y55especies(Tzvelev,2006) nativas de las regiones templadas, con una moderada representación en el este de Asia y Norteamérica y ninguna en el sur de África (Tzvelev, 2006). Su complejidad taxonómica es bien conocida, siendo a veces difícil la delimitación e identificación de sus especies, que se basa sobre todo en caracteres relativos a las piezas de las espiguillas (lema y pálea), por ser frecuente el solapamiento de estos caracteres entre las diferentes especies (Church, 1949; Borril, 1956; Tzvelev, 2006; Whipple et al., 2007; Bushman et al., 2009; Gerlach et al., 2009; Rodionov et al., 2013).

El estudio del material para la revisión del género en el contexto del proyecto Flora iberica, confirma que Glyceria está presente solo en la Península Ibérica donde están representadas 4 especies como ya habían señalado Molina \& Pertiñez (1997), en concreto G. fluitans (L.) R. Br., G. declinata Bréb., G. notata Chevall. y G. spicata Guss., pero los materiales de la última no se ajustan a la variabilidad conocida de $G$. spicata Guss.

G. spicata, descrita de Sicilia (Gussone, 1844), se caracteriza por tener inflorescencias lineares, poco ramificadas y con espiguillas subsésiles, lemas redondeados o truncados, crenados o inconspicuamente dentados, y páleas con ápice entero o emarginado. Plantas con estas características e identificación han sido dadas a conocer en la Península Ibérica como muy frecuentes en Portugal (Coutinho, 1939; Franco \& Rocha-Afonso, 1998) y tan solo en España peninsular para las provincias de Cádiz (Molina \&
Pertiñez, 1997) y Huelva (Talavera, 1987; Molina \& Pertiñez, 1997). Los materiales portugueses estudiados hasta la fecha (COI, LISI y LISU; acrónimos según Thiers, 2019) se corresponden con $G$. declinata, excepto el de una localidad del Bajo Alentejo (LISI 40/2001), que presenta las mismas características del material español. Estas plantas, denominadas como G. spicata presentan, sin embargo, mayores dimensiones del pedúnculo de las espiguillas y menores de las piezas florales y de las anteras que las plantas sicilianas, diferencias a las que aludieron Lambinon \& Deschâtres (1991) y sobre las que se fundamenta la propuesta que se hace a continuación de una nueva subespecie.

Glyceria spicata subsp. onubensis J. López \& Devesa, subsp. nov.

G. fluitans var. spicata sensu Cout., Fl. Portugal ed. 2: 104 (1939), non Guss., FI. Sic. Syn. 2: 784 (1844), pro parte

G. spicata sensu Talavera, FI. Andalucía Occid. 3: 358 (1987), non Guss., FI. Sic. Syn. 2: 784 (1844)

G. spicata sensu Franco \& Rocha-Afonso, Nova Fl. Portugal 3(2): 71 (1998), non Guss., FI. Sic. Syn. 2: 784 (1844), pro parte

Differs from $G$. spicata Guss. subsp. spicata by the smaller dimensions of lemmas [3.5-5(5.3) $\mathrm{mm}$ ], paleas [3.3-4.7(5) $\mathrm{mm}]$, anthers [0.9-1.3(1.5) $\mathrm{mm}$, and pedicel of the lateral spikelets in the main branch of the basal and medium nodes of the inflorescence up to $2.7 \mathrm{~mm}$.

Holotypus: España, Huelva, El Rocío, Arroyo de la Rocina, IV-1978, S. Talavera (SEV 101848).

Descripción: Hierba perenne, rizomatosa, 
acuática o anfibia. Tallos hasta de $65 \mathrm{~cm}$, decumbentes en la base y después ascendentes, con la parte superior por lo general flotante, aplanados, con 2-3 nudos visibles. Hojas con vaina de márgenes soldados en más de la mitad de su longitud, papirácea, estriada, con los nervios papilosos en la cara externa; lígula 5,8-10 mm, membranácea, \pm lacerado-dentada al menos hacia el ápice, y subulada, glabra en la cara externa y \pm retrorso-escabriúscula en la interna; limbo 4,5-17 $\mathrm{cm} \times$ 3-6 $\mathrm{mm}$, linear o estrechamente lanceolado, plano. Panícula 10-30 cm, linear, estrecha, recta, con eje y ramas triquetros, \pm antrorso-escabriúsculos; nudos inferiores con 2-3 ramas, la más larga de 40-110 mm, con (1)2-4(5) espiguillas -rara vez con $1(2)$ ramificaciones con 1 espiguilla- y el resto con 1 espiguilla; nudos medianos con (1)2(3) ramas, la más larga con 1-3(4) espiguillas y la corta con una; nudos superiores con 1 rama uniespiculada. Espiguillas 13-23 mm, \pm cilíndricas, lineares, las laterales de la rama principal de los nudos basales y medios con pedúnculo de $0,5-2,4(2,7) \mathrm{mm}$, con 7-13 flores fértiles y 1 estéril apical reducida a un pequeño lema. Glumas desiguales, la inferior menor que la superior, escariosas -herbáceas en la parte central-, \pm obovadas, uninervias, algo crenadas en el ápice, glabras; la inferior 1,4-2,6(2,8) mm; la superior 2,2-4,2(5) mm. Raquilla con artejos glabros. Lema 3,5-5(5,3) mm, ovado, papiráceo, con el dorso redondeado, y 7 nervios destacados que alcanzan casi el ápice, antrorso-escabriúsculo sobre los nervios, con ápice membranáceo estrecho, obtuso, crenado o inconspicuamente dentado, blanquecino; callo ovado-elíptico, glabro, con reborde cartilaginoso. Pálea 3,3-4,7(5) mm, oblongo-elíptica, casi siempre igualando al lema o apenas superándolo, binervada, biaquillada, entera o emarginada, a veces mucronada, con quillas aladas, finamente antrorso-escabriúsculas. Lodículas 2, carnosillas, soldadas, glabras. Anteras 0,9-1,3(1,5) mm. Cariopsis (1,6)2-2,6 $\times 1-1,4$ $\mathrm{mm}$, oblongo-elipsoidal, con superficie finamente estriado-rugulada, libre.

Fenología: Florece de abril a junio, pudiéndose encontrar plantas florecidas también en febrero y julio.

Distribución y ecología: Península Ibérica, Cerdeña y Marruecos. En España está presente en las provincias de Badajoz, Cádiz, Huelva y Teruel, y en Portugal en el Bajo Alentejo. Habita en orillas de arroyos y lagunas, fuentes, praderas encharcables, charcas temporales y marismas.

Observaciones: $G$. spicata Guss. subsp. spicata presenta lemas de 5,2-5,8 $\mathrm{mm}$, páleas de 5,2-5,6 mm, anteras de 1,7-2,2 $\mathrm{mm}$ y espiguillas laterales de la rama principal de los nudos basales y medianos subsésiles o muy cortamente pedunculadas (pedúnculos de 0,6-1 mm). Su distribución, hasta la fecha, se restringe al $\mathrm{S}$ de Europa (Italia, Grecia y antigua Yugoslavia) y el NW de África (Argelia y Túnez) (Valdés \& Scholz, 2009).

\section{Otros materiales estudiados}

Glyceria spicata subsp. onubensis J. López \& Devesa

ESPAÑA. Badajoz: Alange, laguna Melchor Gómez I, I-174.716/03, 11-VI-2003, B. Sevilla (UNEX 33046). Cádiz: Puerto Real (sin fecha ni recolector) (MA 584986); El Sauzal, 6-VI-1990, A. Galán, J.A. Molina \& S. Sardinero (MAF 164904); de Alcalá a Benalup, al borde de arroyo, sobre sustrato arcillosos, 23-IV-1990, A. Galán, J.A. Molina \& S. Sardinero (MAF 164903); Huelva: Almonte, Reserva Biológica de Doñana, borde de la marisma, 17-V-1972, L. Ramírez (SEV 17695); Almonte, Reserva Biológica de Doñana, laguna del Caño de la Fuente del Duque, 29S QA29, 17-V-1977, n 1805 EV, M. Costa \& E. ValdésBermejo (MA 270953, MA 270957 \& MA 373374); Almonte, Reserva Biológica de Doñana, Caño Fuente del Duque, 29S QA29, 25-VI-1977, n $^{\circ}$ 2310 EV, S. Castroviejo \& E. Valdés-Bermejo (MA 270954); Almonte, Reserva Biológica de Doñana, El Martinazo, 13-V-1966, E.F. Galiano \& J. Novo. (SEV 17699 \& MA 202131); Almonte, Reserva Biológica de Doñaña, El Martinazo, 17-V-1974, B. Cabezudo (SEV 18373 \& SALA 8751); Almonte, Reserva Biológica de Doñana, El Martinazo, 29S QA29, 30-IV-1978, S. Castroviejo \& E. ValdésBermejo (MA 270940); Coto de Doñana, marismas, 21-V-1970, P. Gibbs \& S. Silvestre (SEV 101929); Coto de Doñana, Caño de las Gangas, marisma de la Reserva, S. Rivas (SEV 86460). Hinojos, Palacio del Rey, borde de la marisma, IV-1978, S. Talavera (SEV 101849); Niebla, laguna de los Caballos, 29S QB0136, 15-II-2002, E. Sánchez Gullón \& P. Weickert (MA 827849); Niebla, laguna de los Caballos, 30 S 16976 414042, 28-V-2002, M. Nieto et al. (MGC 52339); Niebla, laguna de Doña Elvira, 30S 17161414461 , borde de laguna, 28-V2002, J.M. Nieto et al. (MGC 52341 \& MGC 53348); Teruel: en lagunica de Tornos, regato de agua casi dulce, 6-VII-1958 (MA 270969); camino de Linares, La Vega de Alcalá de la Selva, prado muy húmedo anegado, $1460 \mathrm{~m}, 10-\mathrm{VII}-1957$, P. Monserrat (MA 270968).

PORTUGAL. Bajo Alentejo: Mértola, Santana de Cambas, monte de costa, charca temporal, C. M. 559, 180 m, 8-V-2001, M.D. Espirito Santo \& P. Arsenio (LISI 40/2001).

MARRUECOS. Larache: Ulad-Mesbah, 4-VI1923, A. Caballero (MA 11761). 
ITALIA. Cerdeña: Monte Rosso, Sos Fungarones, 198 m, 13-VI-2007, S. Bagelle, M.C. Caria \& J.A. Molina (MAF 167085).

\section{Glyceria spicata Guss. subsp. spicata}

ITALIA. Sicilia: Madonie, Margio Scorzone, $37^{\circ} 50^{\prime} \mathrm{N} 14^{\circ} 08^{\prime} \mathrm{E}, 1300-1400 \mathrm{~m}$, marshy area on quartzarenite, 6-VI-1990, F.M. Raimondo et al., 1462 (SALA 153664); Sciare di Santa Venera, 3750'52"N 14오'51'E, 21-IV-2015, J.A. Molina \& P. Minissale, SIC 6 (MAF175163); Cozoffico, $37^{\circ} 14^{\prime} 03^{\prime \prime} \mathrm{N} 15^{\circ} 00^{\prime} 09^{\prime \prime} \mathrm{E}$, en charca temporal, 20IV-2015, J.A. Molina \& P. Minissale, SIC 1 (MAF 175155); Messina, commune di Cesaró, lago Viviere di Cesaró, $37^{\circ} 57^{\prime} \mathrm{N}$ 14 ${ }^{\circ} 42^{\prime} \mathrm{E}, 1250 \mathrm{~m}$, alrededores del lago, 5-VI-2000, C. Aedo et al. (MA 646308).

\section{Agradecimientos}

Al Ministerio de Ciencias e Innovación (proyectos CGL2014-52787-C3-3-P y CGL2017-85204-C3-3-P), cofinanciados por FEDER.

\section{Referencias}

Borril, M. (1956). A biosystematic study of some Glyceria species in Britain. 2. Cytology. Watsonia, 3(6), 299-306.

Bushman, B.S., Sedegui, M. \& Osterbauer, N.K. (2009). Distinguishing Glyceria species of Western North America. Seed Technology, 31(1), 66-75.

Church, G.L. (1949). A cytotaxonomic study of Glyceria and Puccinellia. American Journal of Botany, 36(2), 155-165.

Coutinho, A.X.P. (1939). Flora de Portugal, $2^{a}$ edição (p. 105). Lisboa: Irmãos Bertrand, Lda.

Franco, J.A. \& Rocha-Afonso, M.L. (1998). Nova Flora de Portugal III Graminae, fasc. II (p. 72). Lisboa: Escolar Editora.

Gerlach, J.D., Bushman, B.S., McKay, J.K. \& Meimberg, H. (2009). Taxonomic confusion permits the unchecked invasion of vernal pools in California by low mannagrass (Glyceria declinata). Invasive Plant Science and Management, 2, 92-97.

Gussone, J. (1844). Florae Siculae Synopsis (p. 784). Napoli: Ex Typis Tramater.

Kellogg, E.A. (2015). Flowering plants. Monocots: Poaceae. In K. Kubitzki (Ed.), The Families and Genera of Vascular Plants, 13. Cham: Springer International Publishing.

Lambinon, J. \& Deschâtres, R. (1991) Glyceria notata Chevall. (= Glyceria plicata (Fries) Fries). In D. Jeanmonod \& H.M. Burdet (Eds.), Notes et contributions à la flore de Corse, VII. Candollea, 46(1), 175-226.

Molina, J.A. \& Pertiñez, C. (1997). Aspectos fitogeográficos del género Glyceria R. BR. (Poaceae) en la Península Ibérica. Studia Botanica, 16, 59-81.

Rodionov, A.V., Kotsinyan, A.R., Gnutikov, A.A., Dobroradova, M.A. \& Machs, E.M. (2013). Variability of the ITS1-5.8S rDNA-ITS2 sequence during the divergence of sweet-grass species (Glyceria $\mathrm{R}$. Br.). Russian Journal of Genetics: Applied Research, 3(2): 83-90.

Talavera, S. (1987). Glyceria R. Br. In B. Valdés et al. (Eds.), Flora Vascular de Andalucía Occidental 3 (pp. 357-359). Barcelona: Ketres Editora S.A.

Thiers, B. (2019) Index Herbariorum: A global directory of public herbaria and associated staff. New York Botanical Garden's Virtual Herbarium. Available from: http://sweetgum.nybg.org/science/ih/ [consultado el 28-09-2019].

Tzvelev, N.N. (2006). Synopsis of the mannagrass genus, Glyceria (Poaceae). Botanischeskii Zhurnal (Moscow and Leningrad), 91(2), 255-276.

Valdés, B. \& Scholz, H.; with contributions from E. von Raab-Straube \& G. Parolly (2009). Poaceae (pro parte majore). Euro+Med Plantbase - the information resource for Euro-Mediterranean plant diversity. http:// ww2.bgbm.org/EuroPlusMed/ [consultado el 28-092019].

Whipple, I.G., Barkworth, M.E. \& Bushman, B.S. (2007). Molecular insights into the taxonomy of Glyceria (Poaceae: Meliceae) in North America. American Journal of Botany, 94(4), 551-557. 